\title{
Management of the positive axilla in 2017
}

\author{
Eli Avisar ${ }^{1}$ (i) Tolga Ozmen ${ }^{1}$
}

Received: 3 March 2017/Accepted: 29 March 2017/Published online: 5 April 2017

(C) Springer Science+Business Media New York 2017

One of the greatest advances in breast cancer surgical management over the last few years has been the more conservative management of the axilla.

After acceptance of the Halstedian paradigm, axillary dissection (AD) became the "one size fits all" treatment modality for all stages of breast cancer. This approach was first challenged in the late 80 's by Silverstein et al. in ductal carcinoma in situ (DCIS) patients [1]. His report showing no axillary metastasis in any of the DCIS patients led to the omission of AD for DCIS patients.

Subsequent large trials reported that although axillary metastases can be found in $40 \%$ of clinically node-negative patients, only $15 \%$ of them became clinically perceptible if left untreated [2]. Widespread use of screening mammography in the 90's led to further decrease in the rate of axillary positivity to $22 \%$ [3]. According to this calculation, $78 \%$ of early-stage breast cancer patients were undergoing unnecessary $\mathrm{AD}$ associated with a $16 \%$ risk of lymphedema [4]. Two studies investigated the possible omission of $\mathrm{AD}$ in elderly ( $>60$ years old) patients revealing similar long-term outcomes with and without $\mathrm{AD}$ $[5,6]$.

$\mathrm{AD}$, however, was still the treatment of choice for a clinically positive axilla and remained an essential staging procedure enabling stratification of patients, who would benefit from adjuvant therapy. In the 90's, axillary staging was replaced by sentinel lymph node biopsy (SLNB) after this technique was validated in many studies including the

Eli Avisar

eavisar@med.miami.edu

1 Department of Surgery/Surgical Oncology, Miller School of Medicine, University of Miami, Miami, FL, USA
MILAN, NSABP-B32, and ALMANAC trials [7-9] limiting the use of $\mathrm{AD}$ for positive sentinel nodes only.

The next important advance was the omission of $\mathrm{AD}$ even in the presence of some axillary disease. The ACOSOG-Z0011 and AMAROS trials recruited patients with limited macrometastasis to the axilla $[10,11]$. ACOSOGZ0011 included patients treated with lumpectomy and adjuvant radiation therapy reporting no additional benefit in regional control of the axilla for completion $\mathrm{AD}$ in this specific group of patients with low recurrence risk (0.9 vs. $0.5 \%, p>0.05$ ). AMAROS trial compared treating SLNB positive patients with $\mathrm{AD}$ versus radiation treatment to the axilla. This trial also reported no additional benefit of $\mathrm{AD}$ compared to axillary radiation (RT) in DFS (86.9\% in the $\mathrm{AD}$ group vs. $82.7 \%$ in the $\mathrm{RT}$ group, $p=0.18)$. $\mathrm{AD}$, however, was associated with an approximately two-fold increase in clinical signs of lymphedema.

In parallel, the combination of SLNB and neo-adjuvant chemotherapy (NAC) was studied as well. Some proposed that the sentinel node biopsy should be performed before the chemotherapy, and some argued that it should be postponed to after the treatment. That very question was studied in a large single institutional study from MD Anderson comparing SLNB before and after NAC [12]. Identification rates (IR) and false negative rates (FNR) for SLNB before and after NAC were similar [98.7 vs. $97.4 \%$ ( $p=0.017)$ and 4.1 vs. $5.9 \%(p=0.39)$, respectively]. Additional multicenter studies also reported concordant results. In a retrospective analysis of NSABP B-27 data, performance of SLNB after NAC was associated with an IR of $85 \%$ and a FNR of $11 \%$ [13]. Use of radionuclide decreased FNR to $9 \%$ in comparison to $14 \%$, which was achieved when only lymphazurin was used $(p=0.5)$. The second multicenter study was the prospectively conducted GANEA study, which reported an IR of $90 \%$ and FNR of 
$11.5 \%$ for SLNB after NAC [14]. When the results of NSABP-B27 and GANEA studies were combined, an IR of $86.5 \%$ and a FNR of $10.9 \%$ were found [15]. Two metaanalyses assessed the accuracy of SLNB after NAC. The first study summarized 21 studies/1273 patients and found an IR of $90 \%$ and a FNR of $12 \%$ [16]. The second study summarized 24 studies/1799 patients and found an IR of $89.6 \%$ and a FNR of $8.4 \%$ [17]. Still yet, performing a SLNB after NAC was limited to patients who did not have a clinically positive axilla prior to treatment. Those who did have a positive axilla before NAC were treated with a mandatory AD.

The following logical step was to study whether a previously positive axilla that down-staged and became negative after NAC could be reliably staged with a SLNB alone after NAC. This question was investigated in three recent separate studies, ACOSOG-Z1071, SENTINA, and SN-FNAC, in which SLNB was followed by confirmatory AD [18-20]. In all three studies, removing $\geq 2$ sentinel nodes and combining radionuclide tracer with blue dye increased sensitivity and decreased FNR. In the ACOSOGZ1071 study, however, 1/3 of the patients had a clip placed at the time of the biopsy and before NAC in the positive axillary node. When the clipped node was identified, and excised during SLNB, the FNR decreased to $6.8 \%$ but when the clipped node was not found, the FNR was as high as $39 \%$. These findings led to different complicated techniques described to re-localize the clipped node after NAC. Regardless, those three studies were summarized in two reviews. The first review involved 2471 patients and found an IR of $89 \%$ and a FNR of $14 \%$ [21]. According to this review usage of IHC decreased FNR to $8.7 \%$, while without IHC, FNR was $16 \%$. The second review included 3398 patients and reported a pCR of $39.2 \%$, an IR of $90.9 \%$ and a FNR of $13 \%$ [22]. Long-term clinical outcomes of SLNB alone for down-staged axilla after NAC has not however been reported yet.

The coming years might bring even more changes in the management of the positive axilla. The ALLIANCE A011202 trial (https://clinicaltrials.gov/ct2/show/ NCT01901094) is now recruiting patients with T1-3/N1/ M0 at presentation and who became clinically negative after NAC. These patients undergo SLNB and if SLNB is positive get randomized to AD vs. RT.

Those very significant changes in management, however, bring also new clinical challenges to the multidisciplinary team trying to decide about the most appropriate treatment course.

First, it becomes extremely important to correctly stage the axilla at presentation. Missing the presence of a positive metastatic axillary node might lead to a decision of surgery first which will result in a positive sentinel node that might not be appropriate for observation only and could end up in AD or RT that could have been avoided by NAC.

Second, the mere identification of axillary disease is not necessarily an indication for NAC. That would be especially true in a small luminal A or B tumor that otherwise might not need chemotherapy even after surgery and, furthermore, might have very small chances to lead to a complete response in the axilla. The different molecular signature available at the present time is focusing on survival or recurrence. New molecular signatures need to be developed that can accurately predict the nodal response to chemotherapy in order to correctly guide the patients.

How to reassess the axilla after NAC is a challenging issue as well. Since even the presence of micrometastasis after NAC is predictive of residual axillary disease in up to $98 \%$ of the patients [22], the most accurate reassessment of the axilla for residual disease would minimize the risk of an unplanned AD concomitant or subsequent to the SLNB.

In our institution, we have implemented and are currently studying a systematic sonographic axillary staging at presentation, preferentially by the surgeons. Needle biopsies are then preformed of suspicious nodes. At completion of NAC, the axilla is again systematically staged sonographically, taking into account the pre-treatment disease locations. Any residual morphologically suspicious node undergoes a repeat needle biopsy. If there is evidence of residual disease, the patient is scheduled for $\mathrm{AD}$. If there is no detectable residual disease, the patient is scheduled for a SLNB.

Prediction of likelihood of a certain metastatic axillary disease to fully respond to NAC is done currently based on the following criteria.

(a) The indication for chemotherapy in the adjuvant setting. If a patient will need chemotherapy anyway in the adjuvant setting and metastatic axillary disease is identified, NAC will be offered to the patient in an attempt to avoid AD.

(b) The molecular signature of the tumor. For those patients who would not necessarily need chemotherapy, we use some of the available molecular signatures in an attempt to be predictive about the axillary response but further refinement is necessary. Furthermore, the currently published information regarding preoperative use of hormonal modulation is insufficient to accurately predict a complete axillary response. Hopefully, further refinement in the molecular signature assays will help better manage the positive axilla.

Acknowledgements There is no sponsoring organization and no financial relationship to report.

Compliance with ethical standards

Conflict of interest There are no conflicts of interest. 


\section{References}

1. Silverstein MJ, Cohlan BF, Gierson ED et al (1992) Duct carcinoma in situ: 227 cases without microinvasion. Eur J Cancer 28:630-634

2. Fisher B, Montague F, Redmond C et al (1985) Ten-year results of a randomized trial comparing radical mastectomy and total mastectomy with or without radiation. $\mathrm{N}$ Engl $\mathrm{J}$ Med 312:674-681

3. Hofvind S, Lee CI, Elmore JG (2012) Stage-specific breast cancer incidence rates among participants and non-participants of a population-based mammographic screening program. Breast Cancer Res Treat 135:291-299

4. McLaughlin SA, Wright MJ, Morris KT et al (2008) Prevalence of lymphedema in women with breast cancer 5 years after sentinel lymph node biopsy or axillary dissection: objective measurements. J Clin Oncol 26:5213-5219

5. International Breast Cancer Study Group (2006) Randomized trial comparing axillary clearance versus no axillary clearance in older patients with breast cancer: first results of international breast cancer study group trial 10-93. J Clin Oncol 24:337-344

6. Martelli G, Boracchi P, Ocenti A et al (2014) Axillary dissection versus no axillary dissection in older T1N0 breast cancer patients: 15 year results of trial and out-trial patients. Eur J Surg Oncol 40:805-812

7. Veronesi U, Paganelli G, Viale G et al (2003) A randomized comparison of sentinel-node biopsy with routine axillary dissection in breast cancer. N Engl J Med 349:546-553

8. Krag DN, Anderson SJ, Julian TB et al (2010) Sentinel-lymphnode resection compared with conventional axillary-lymph-node dissection in clinically node-negative patients with breast cancer: overall survival findings from the NSABP B-32 randomised phase 3 trial. Lancet Oncol 11:927-933

9. Mansel RE, Fallowfield L, Kissin M et al (2006) Randomized multicenter trial of sentinel node biopsy versus standard axillary treatment in operable breast cancer: the ALMANAC Trial. J Natl Cancer Inst 98:599-609

10. Giuliano AE, Hunt K, Ballman K et al (2011) Axillary dissection vs no axillary dissection in women with invasive breast cancer and sentinel node metastases: a randomized clinical trial. JAMA 305:569

11. Donker M, van Tienhoven G, Straver ME et al (2014) Radiotherapy or surgery of the axilla after a positive sentinel node in breast cancer (EORTC 10981-22023 AMAROS): a randomized multicentre, open-label, phase 3 non-inferiority trial. Lancet Oncol 15:1303-1310
12. Hunt KK, Yi M, Mittendorf EA et al (2009) Sentinel lymph node surgery after neoadjuvant chemotherapy is accurate and reduces the need for axillary dissection in breast cancer patients. Ann Surg 250:558-566

13. Mamounas EP, Brown A, Anderson S et al (2005) Sentinel node biopsy after neoadjuvant chemotherapy in breast cancer: results from National Surgical Adjuvant Breast and Bowel Project Protocol B-27. J Clin Oncol 23:2694-2702

14. Classe JM, Bordes V, Campion L et al (2009) Sentinel lymph node biopsy after neoadjuvant chemotherapy for advanced breast cancer: results of Ganglion Sentinelle et Chimiotherapie Neoadjuvante, a French prospective multicentric study. J Clin Oncol 27:726-732

15. Mamounas EP (2015) Impact of neoadjuvant chemotherapy on locoregional surgical treatment of breast cancer. Ann Surg Oncol 22:1425-1433

16. Xing Y, Foy M, Cox DD et al (2006) Meta-analysis of sentinel lymph node biopsy after preoperative chemotherapy in patients with breast cancer. Br J Surg 93:539-546

17. Kelly AM, Dwamena B, Cronin P et al (2009) Breast cancer sentinel node identification and classification after neoadjuvant chemotherapy-systematic review and meta analysis. Acad Radiol $16: 551-563$

18. Boughey JC, Suman VJ, Mittendorf EA et al (2013) Sentinel lymph node surgery after neoadjuvant chemotherapy in patients with node-positive breast cancer: theACOSOGZ1071 (Alliance) clinical trial. JAMA 310:1455-1461

19. Kuehn T, Bauerfeind I, Fehm T et al (2013) Sentinel-lymph-node biopsy in patients with breast cancer before and after neoadjuvant chemotherapy (SENTINA): a prospective, multicenter cohort study. Lancet Oncol 14:609-618

20. Boileau JF, Poirier B, Basik M et al (2015) Sentinel node biopsy after neoadjuvant chemotherapy in biopsy-proven node-positive breast cancer: the SN FNAC study. J Clin Oncol 33(3):258-264

21. Fu JF, Chen HL, Yang J, Yi CH, Zheng S (2014) Feasibility and accuracy of sentinel lymph node biopsy in clinically node-positive breast cancer after neoadjuvant chemotherapy: a meta-analysis. PLoS ONE 9(9):e105316. doi:10.1371/journal.pone. 0105316 eCollection 2014

22. El Hage Chehade H, El Tokhy O, Heeney J, Kasem A, Mokbel K (2016) Is sentinel lymph node biopsy a viable alternative to complete axillary dissection following neoadjuvant chemotherapy in women with node-positive breast cancer at diagnosis? An updated meta-analysis involving 3,398 patients. Am J Surg 212(5):969-981 